\title{
Оптимизация условий насыщения и предподготовки хромато-десорбционных микросистем для получения газовых смесей ацетона
}

\author{
(c) 2020 Колесниченко И.Н., Аникина М.А., Платонов И.А. \\ Самарский наџионально-исследовательский университет имени академика С.П. Королева, Самара
}

Поступила в редакцию 17.07.2020 г.

DOI: $10.17308 /$ sorpchrom.2020.20/2949

Анализ выдыхаемого воздуха является активно развивающейся областью неинвазивной медицинской диагностики. Такой подход позволяет изучать динамику физиологических процессов в организме человека, а также выявить патологию на ранней стадии развития. Однако широкое применение неинвазивных методов сдерживается несовершенством методик пробоотбора и пробоподготовки, приводящее ко внесению ощутимых погрешностей в результаты.

Цель настоящей работы - разработка устройств, способов и методических приёмов повышения точности неинвазивного количественного определения ацетона в выдыхаемом воздухе для неинвазивной диагностики сахарного диабета. Предметом исследования являлись устройства, использование которых на стадиях градуировки и подготовки проб позволяет снизить суммарную погрешность анализа.

В качестве проточного контейнера для хромато-десорбционной микросистемы (ХДмС) были выбраны инъекционные иглы, заполненные сорбентом. В качестве сорбентов использовались инертный носитель Chromaton N-AW, модифицированный 25\% сорбционно-активной неорганической солью $\mathrm{CoCl}_{2}$; инертное волокно, покрытое $25 \%$ полиэтиленгликолем (марка ПЭГ-20М). Насыщение ХДмС осуществлялось потоковым и капельным методами при температурах 25 и $50^{\circ} \mathrm{C}$. Десорбция аналита осуществлялась при 70 и $100^{\circ} \mathrm{C}$ дискретным пропусканием 0.5 см$^{3}$ инертного газа (азота) через ХДмС, помещенную в термодесорбер, совмещенный с испарителем газового хроматографа.

Полученные в ходе исследования изготовленных ХДмС данные указывают на преимущество капельного метода насыщения перед потоковым, поскольку в этом случае возрастает количество дискретных вводов в одном цикле работы, за счет чего обеспечивается поддержание постоянства концентрации аналита в газовой смеси. Показано, что за счет регулирования условий предподготовки системы (подбор техники насыщения и десорбции) можно получать газовые смеси с заданным содержанием ацетона. Обоснована возможность проведения многоточечных калибровок при использовании ХДмС. Различные концентрации градуировочных газовых смесей достигаются путем изменения температуры десорбции и посредством регулирования условий предварительной подготовки системы объема пропускаемого инертного газа или газовой смеси ацетона. Экспериментально установлено, что для насыщения ХДмС оптимальным является капельный метод, объем дозирования 1 мкл, и предварительную подготовку систем целесообразно проводить при $25^{\circ} \mathrm{C}$ дискретно со скоростью $0.5 \mathrm{~cm}^{3} /$ мин. Условия десорбции могут варьироваться от 70 до $100^{\circ} \mathrm{C}$, время продувки определяется ступенью десорбции. При работе с системами, содержащими $\mathrm{CoCl}_{2}$, повышение температуры выше $100^{\circ} \mathrm{C}$ нецелесообразно.

Экспериментально установлено, что при дискретном дозировании в получаемых газовых смесях погрешность поддержания стабильности $\Delta$ варьируется в пределах $10-15 \%$, что позволяет повысить точность анализа устройств с использованием ХДмС.

Полученные в ходе эксперимента закономерности и разработанные методики могут быть использованы при разработке устройства для экспрессного количественного определения ацетона в выдыхаемом воздухе и повышении его чувствительности как к данному биомаркеру, так и к другим.

Ключевые слова: газовая хроматография, неинвазивная диагностика, биомаркеры, хроматодесорбционные системы, концентрирование. 


\section{Введение}

Согласно официальной статистке Всемирной организации здравоохранения (BO3) [1], число заболевших сахарным диабетом по всему миру растет с каждым годом, поэтому ранняя диагностика этого заболевания является актуальной задачей. Перспективным решением является неинвазивная диагностика состояния здоровья по составу выдыхаемого воздуха. Современные тенденции развития безопасных исследований и щадящих приемов забора пробы, исключающих физический и эмоциональный дискомфорт пациента, предъявляют высокие требования к разрабатываемым медицинским приборам и методикам проведения диагностики [2]. В настоящее время экспресс-устройства, способные быстро и безболезненно проводить диагностику заболеваний желудочно-кишечного тракта, дыхательной, пищеварительной и сердечно-сосудистых систем, уже существуют [3-5], но не введены в массовое производство по причине несовершенства методик пробоотбора и пробоподготовки, приводящих к внесению основных погрешностей в результаты анализа.

Основной проблемой на сегодняшний день остаётся отсутствие оптимизированных высокочувствительных и высокоточных методик, позволяющих количественно определять содержание специфических следовых концентраций летучих соединений в альвеолярном воздухе, и адекватных (идентичных друг другу) методик пробоотбора и пробоподготовки, исключающих внесение дополнительных артефактов $[6,7]$.

Цель настоящей работы - разработка устройств, способов и методических приёмов повышения точности неинвазивного количественного определения ацетона в выдыхаемом воздухе.

\section{Экспериментальная часть}

Для достижения цели необходим выбор целесообразной модели изготовления хромато-десорбционных систем инъекционного типа, подготовка сорбентов, нанесение микро- и нанопленок на инертные носители, экспериментальное определение оптимальных условий насыщения и предварительной подготовки хроматодесорбционных систем инъекционного типа.

В качестве проточного контейнера для хромато-десорбционной микросистемы (ХДмС) были использованы инъекционные иглы внутренний диаметр 0.5 мм, длина 40 мм, заполненные сорбентами, представленные на рисунке 1, на входе и выходе устанавливались инертные газопроницаемые заглушки, для предотвращения высыпания сорбента во время использования системы $[8,9]$.

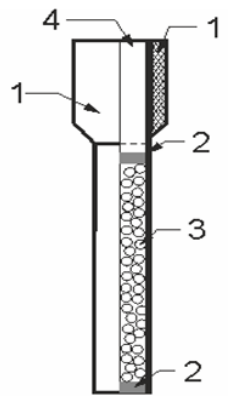

Рис. 1. Принципиальная схема (ХДмС):

1 - головка иглы, 2 - заглушка, 3 - сорбент, 4 - внутренний канал иглы

Fig. 1. The CDmS scheme:

1 - needle head, 2 - plug, 3 - sorbent, 4 - channel inside the needle 
В качестве сорбентов использовались: инертный носитель Chromaton N-AW, модифицированный $25 \%$ сорбционно-активной неорганической солью $\mathrm{CoCl}_{2}$; инертное волокно, покрытое 25\% полиэтиленгликолем (марка ПЭГ-20М). Выбор сорбента обусловлен тем, что непористые соли, до недавнего времени используемые только для газохроматографического разделения, перспективны в качестве сорбента для концентрирования микроколичеств ЛОС [10]. Сорбционно-активные вещества наносились на инертный носитель, что обеспечивает уменьшение времени диффузии аналита вглубь зерна сорбента, которая является лимитирующей стадией десорбции, и позволяет уменьшить время установления равновесия в системе при дискретном режиме работы. ПЭГ-20М выбран как система сравнения, широко применяемый для разделения газовых смесей кетонов и альдегидов, в том числе ацетона, для рабочих температур $\left.70-250^{\circ} \mathrm{C}\right)[11]$.

Суть хромато-десорбционного способа заключается в равновесном насыщении летучим органическим веществом потока инертного газа при его прохождении через трубчатую проточную систему, заполненную сорбентом, предварительно насыщенную аналитом. Насыщение ХДмС известным количеством аналита осуществлялось двумя способами: 1) пропусканием потока газа, содержащего известное избыточное количество аналита через ХДмС со скоростью $0.5 \mathrm{~cm}^{3} /$ мин до тех пор, пока концентрация аналита на входе и выходе из системы не станут одинаковыми (т.е. до «проскока») [12-14]; 2) капельным методом, заключающимся в нанесении 1 мкл ацетона и пропускании $5 \mathrm{~cm}^{3}$ азота со скоростью $0.5 \mathrm{~cm}^{3} /$ мин при температуре $25^{\circ} \mathrm{C}$ для распределения аналита в порах сорбента. Закономерности хроматодесорбционного процесса описывает теория методов адсорбционного равновесного концентрирования [6, 12-13, 15].

Десорбция аналита осуществлялась при $100^{\circ} \mathrm{C}$ дискретным пропусканием $0.5 \mathrm{~cm}^{3}$ инертного газа (азота) через ХДмС, помещенную в термодесорбер, совмещенный с испарителем газового хроматографа.

\section{Обсуждение результатов}

В таблице 1 представлены характеристики изготовленных ХДмС, насыщение которых осуществлялось при температурах 25 и $50^{\circ} \mathrm{C}$.

Таблица 1. Характеристики исследуемых хромато-десорбционных микросистем инъекционного типа при температуре десорбции $70^{\circ} \mathrm{C}$

Table 1. Characteristics of the studied chromato-desorption microsystems of injection type at the desorption temperature of $70^{\circ} \mathrm{C}$

\begin{tabular}{|c|c|c|c|c|c|}
\hline Сорбент & $\begin{array}{c}\text { Масса } \\
\text { сорбента, мг }\end{array}$ & \multicolumn{2}{|c|}{$\begin{array}{l}\text { Концентрация ацетона } \\
\text { при десорбции, } 10^{-4} \text { мг }\end{array}$} & $\begin{array}{c}\text { Количество } \\
\text { стабильных } \\
\text { дискретных вводов N } \\
\text { при отклонении } \\
\Delta<15 \%\end{array}$ & $\begin{array}{c}\text { Возобнов- } \\
\text { ляемый } \\
\text { ресурс, } \\
\text { единиц раз }\end{array}$ \\
\hline 1 & 2 & \multicolumn{2}{|l|}{3} & 4 & 5 \\
\hline \multicolumn{6}{|c|}{ Насыщение потоком газа } \\
\hline \multicolumn{6}{|c|}{ Температура насыщения $25^{\circ} \mathrm{C}$} \\
\hline \multirow{2}{*}{$\begin{array}{l}\mathrm{CoCl}_{2} \text { на } \\
\text { Chromaton }\end{array}$} & \multirow{2}{*}{3.3} & Ступень I & 0.5 & 2 & \multirow{2}{*}{0} \\
\hline & & Ступень II & 0.2 & 3 & \\
\hline \multirow{2}{*}{$\begin{array}{l}\text { ПЭГ на } \\
\text { волокне }\end{array}$} & \multirow{2}{*}{2.2} & Ступень I & 1.7 & 6 & \multirow{2}{*}{5} \\
\hline & & Ступень II & 0.8 & 7 & \\
\hline
\end{tabular}

Колесниченко и др. / Сорбционные и хроматографические процессы. 2020. Т. 20. № 4. С.426-433 


\begin{tabular}{|c|c|c|c|c|c|}
\hline \multicolumn{6}{|c|}{ Температура насыщения $50^{\circ} \mathrm{C}$} \\
\hline 1 & 2 & \multicolumn{2}{|l|}{3} & 4 & 5 \\
\hline \multirow{2}{*}{$\begin{array}{l}\mathrm{CoCl}_{2} \text { на } \\
\text { Chromaton }\end{array}$} & \multirow{2}{*}{3.3} & Ступень I & 0.2 & 2 & \multirow{2}{*}{0} \\
\hline & & Ступень II & - & - & \\
\hline \multirow{2}{*}{$\begin{array}{l}\text { ПЭГ на } \\
\text { волокне }\end{array}$} & \multirow[b]{2}{*}{2.2} & Ступень I & 0.8 & 6 & \multirow{2}{*}{5} \\
\hline & & Ступень II & 0.2 & 6 & \\
\hline \multicolumn{6}{|c|}{ Капельный метод } \\
\hline \multicolumn{6}{|c|}{ Температура насыщения $25^{\circ} \mathrm{C}$} \\
\hline \multirow{2}{*}{$\begin{array}{l}\mathrm{CoCl}_{2} \text { на } \\
\text { Chromaton }\end{array}$} & \multirow{2}{*}{3.3} & Ступень I & 0.6 & 4 & \multirow{2}{*}{0} \\
\hline & & Ступень II & 0.3 & 7 & \\
\hline \multirow{2}{*}{$\begin{array}{l}\text { ПЭГ на } \\
\text { волокне }\end{array}$} & \multirow{2}{*}{2.2} & Ступень I & 2.1 & 7 & \multirow{2}{*}{5} \\
\hline & & Ступень II & 1.0 & 8 & \\
\hline \multicolumn{6}{|c|}{ Температура насыщения $50^{\circ} \mathrm{C}$} \\
\hline \multirow{2}{*}{$\begin{array}{l}\mathrm{CoCl}_{2} \text { на } \\
\text { Chromaton }\end{array}$} & \multirow{2}{*}{3.3} & Ступень I & 0.4 & 3 & \multirow{2}{*}{0} \\
\hline & & Ступень II & 0.1 & 2 & \\
\hline \multirow{2}{*}{$\begin{array}{l}\text { ПЭГ на } \\
\text { волокне }\end{array}$} & \multirow{2}{*}{2.2} & Ступень I & 1.3 & 6 & \multirow{2}{*}{4} \\
\hline & & Ступень II & 0.8 & 7 & \\
\hline
\end{tabular}

Как видно из представленных данных, капельный метод позволяет осуществлять более эффективное насыщение, поскольку в этом случае при эксплуатации ХДмС возрастает количество дискретных вводов. Этот факт хорошо согласуется с ранее полученными данными для гетерогенных сорбционных макросистем. Известно [16-20], что в гетерогенных системах путем создания $25 \%$ избытка концентрации в начальной части системы обеспечивается буферный эффект. Сущность этого эффекта заключается в подпитки последней части системы, за счет чего обеспечивается поддержание постоянства концентрации аналита в газовой смеси.

При дискретном режиме ресурс работы хромато-десорбционной микросистемы, наполненной волокном, модифицированным ПЭГ 20М, составляет не менее 6 последовательных циклов, при этом возобновляемый ресурс системы - не менее 4. Однако системы с $\mathrm{CoCl}_{2}$ после одного цикла работы становятся непригодны для дальнейшего использования, так как специфические свойства сорбента приводят к спеканию гранул и затруднению прохождения потока газа.

На рис. 2 представлен общий вид графической зависимости концентрации ацетона в газовой фазе на выходе из ХДмС от количества пропущенного газа.

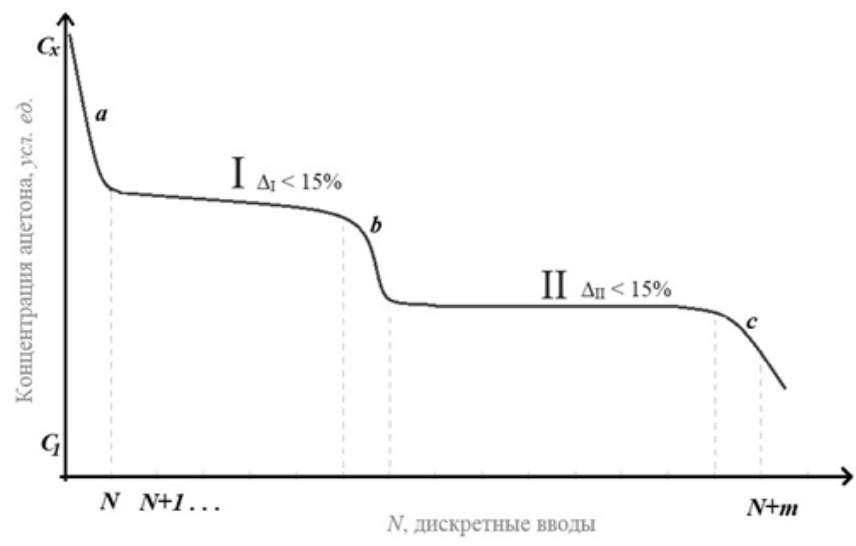

Рис. 2. Общий вид графической зависимости концентрации ацетона в газовой фазе на выходе из ХДмС от количества пропущенного газа

Fig. 2. Dependence diagram of the concentration of acetone in the gas phase at the CDmS's output on the amount of the blown gas 
Десорбционная кривая имеет полиномиальный характер, вследствие чего создается возможность проведения многоточечных калибровок путем не только изменения температуры десорбции, но и регулирования условий предварительной подготовки системы. Линейные участки (ступени I и II на рис. 2) отражают равновесное состояние системы и соответствуют газовой смеси со стабильным содержанием ацетона, погрешность поддержания стабильности $\Delta$ варьируется в пределах 10-15\%. Экспоненциальные участки a, b, с отражают неравновесное состояние системы. Исходя из десорбционной кривой, можно выбрать условия предподготовки таким образом, чтобы получить смесь с заданной концентрацией ацетона, соответствующей ступени I или II. При этом температура десорбции не изменяется [21].

Таким образом, условия предподготовки выбираются, чтобы два первых ввода соответствовали участку неравновесного состояния системы (участок а) перед началом ступени I если необходимо получить смесь с большей концентрацией ацетона, и неравновесного состояния системы (участок b) перед началом ступени II если необходимо получить смесь с большей концентрацией ацетона.

На рисунке 3 представлена графическая зависимость концентрации ацетона в газовой фазе от количества пропущенного инертного газа ( каждый дискретный ввод соответствует пропусканию $0.5 \mathrm{~cm}^{3}$ инертного газа), полученная при температуре десорбции $70^{\circ} \mathrm{C}$.

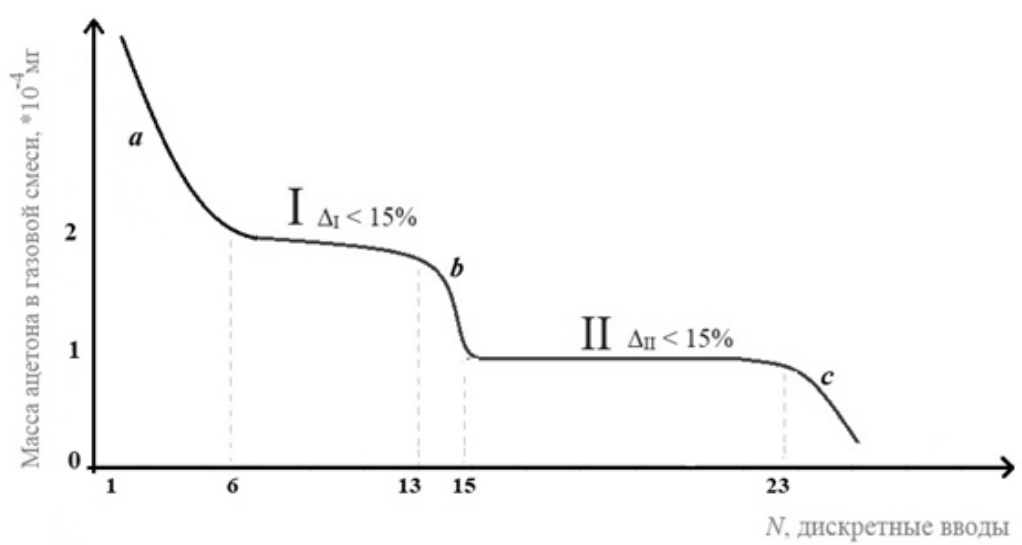

Рис. 3. Графическая зависимость концентрации ацетона в газовой фазе от количества пропущенного инертного газа (каждый дискретный ввод соответствует пропусканию $0.5 \mathrm{~cm}^{3}$ инертного газа), полученная при температуре десорбции $70^{\circ} \mathrm{C}$ для системы ХДмС, заполненной инертным волокном, с нанесенным 20\% ПЭГ 20М

Fig. 3. Dependence diagram of the concentration of acetone in the gas phase on the amount of blown gas (each discrete input corresponds to $0.5 \mathrm{ml}$ blowing with inert gas), obtained at the desorption temperature of $70^{\circ} \mathrm{C}$ for the $\mathrm{CDmS}$ filled with inert fibre covered with $20 \%$ PEG-20M

Из графика следует, что при необходимости получения концентрации, соответствующей ступени I, предподготовка системы будет заключаться в пропускании $3 \mathrm{~cm}^{3}$ инертного газа при $\mathrm{T}=70^{\circ} \mathrm{C}$, что соответствует 6 дискретным циклам, а при получении газовой смеси с содержанием ацетона, соответствующим ступени II $-7 \mathrm{~cm}^{3}$ (15 дискретных циклов). Необходимо отметить, что чем ниже температура десорбции, тем больше продолжительность постоянства содержания аналита в газовой фазе при дискретном дозировании и больше стабильность состава $\Delta$. Полученные закономерности согласуются с ранее установленным аналогичным зависимостям для полибарботажного и хромато-десорбционного способов получения газовых смесей ЛОС и сероводорода, однако при значительном ресурсе таких макросистем (6-80 часов 
непрерывной работы при скорости пропускания инертного газа $1 \mathrm{~cm}^{3} / \mathrm{Mин)} \mathrm{этот} \mathrm{эф-}$ фект не нашел большого практического применения, в то время как при работе с микросистемами ХДмС инъекционного типа этап предподготовки системы значительно сокращается и не отличается от рабочего режима системы.

\section{Заключение}

В ходе работы был произведён выбор целесообразной модели ХДмС инъекционного типа и изготовлены опытные образцы, изготовлены и изучены два вида сорбентов: инертное волокно, с нанесенной $25 \%$ неподвижной жидкой фазой полиэтиленгликоль марки ПЭГ-20М и инертный носитель Chromaton N-AW, модифицированный $25 \%$ сорбционно-активной неорганической солью $\mathrm{CoCl}_{2}$. Экспериментально определены оптимальные условия насыщения и предварительной подготовки ХДмС: предварительную подготовку целесообразно проводить при $25^{\circ} \mathrm{C}$ капельным методом (объем аналита 1 мкл; объем пропускаемого инертного газа $5 \mathrm{~cm}^{3}$ ), условия десорбции могут варьироваться от 70 до $100^{\circ} \mathrm{C}$. При работе с системами, содержащими $\mathrm{CoCl}_{2}$, повышение температуры выше $100^{\circ} \mathrm{C}$ нецелесообразно.

Полученные в ходе эксперимента данные и разработанные методики могут быть использованы при разработке усовершенствованного устройства для экспрессного

количественного определения ацетона в выдыхаемом воздухе и повышении его чувствительности как к данному биомаркеру, так и к другим.

Работа выполнена при поддержке Минобрнауки России в рамках государственного задания на выполнение работ (проект № 4.6875.2017/8.9).

\section{Список литературы}

1 Всемирная организация здравоохранения. Глобальный доклад по диабету. Женева. $2018.88 \mathrm{c}$.

2 Horvath I., Barnes P.J., Loukides S., Sterk P.J. et al. // European Respiratory Journal. 2017. Vol. 49, No 4, pp. 1600965.

3 Su M., Ge L., Kong Q., Zheng X. et al. // Biosensors and Bioelectronics. 2015. Vol. 63. pp. 232-239.

4 Barberis A., Garbetta A., Cardinali A., Bazzu G. et al. // Biosensors and Bioelectronics. 2017. Vol. 88. pp. 159-166.

5 Chung J.-H., Lee KE, Nam C.-W., Doh J.H. et al. // The American Journal of Cardiology. 2017. Vol. 120. No 3. pp. 362-368.

6 Phillips M. // Scientific American. 1992. Vol. 267. No 3. pp. 74-79.

7 Вакс В.Л., Домрачева Е.Г., Собакинская Е.А., Черняева М.Б. // Успехи физических наук. Приборы и методы исследования. 2014. T. 184. № 7. C. 739-758.

8 Soury S., Bahrami Ab., Alizadeh S., Shahna F.G. et al. // Microchemical Journal. 2019. Vol. 148. pp. 346-354.
9 Heidari M., Bahrami Ab., Ghiasvand A.R., Shahna F.G. et al. // Analytica Chimica Acta. 2013. Vol. 785. pp. 67-74.

10 Журавлева Г.А. Дисс. канд. хим. наук. Санкт-Петербург. 2014. 102 с.

11 Лурье А.А. Хроматографические материалы. М. Химия. 1978. 440 с.

12 Платонов И.А., Колесниченко И.Н., Платонов В.И., Лобанова М.С. и др. // Coвременная наука: актуальные проблемы $и$ пути их решения. 2016. №. 1. С. 41-46.

13 Платонов И.А., Колесниченко И.Н., Новикова Е.А., Павлова Л.В и др. // Измерительная техника. 2017. № 8. С. 67-70.

14 Forina M. // Annali di Chemica. 1975. Vol. 65. pp. 491-508.

15 Vitenberg A.G. // Journal of Chromatography. 1991. Vol. 556. pp. 1-24.

16 Platonov I.A., Kolesnichenko I.N., Lange P.K. // Measurement Techniques. 2017. Vol. 59. No 12. pp. 1330-1333.

17 Березкин В.Г., Платонов И.А., Смыгина И.Н. // Химия и химическая технология. 2007. T. 50. № 8. C. 22-24.

18 Платонов И.А., Исмагилов Д.Р., Кудряшов С.Ю., Смыгина И.Н. и др. // Журнал 
аналитической химии. 2006. Т. 61. № 1. С. 59-64.

19 Витенберг А.Г., Конопелько Л.А. // Журнал аналитической химии. 2011. Т. 66. №5. C. 452-472.
20 Платонов И.А. // Сорбционные и хроматографические прочессы. 2006. Т. 6. № 5. С. 833-843.

21 Forina M., Lanteri S., Casale M. // Journal of Chromatography A. 2007. Vol. 1158. pp. 6193.

\title{
Optimisation of the conditions for the saturation and preparation of chromato-desorption microsystems for the production of acetone gas mixtures
}

\author{
(C) 2020 Kolesnichenko I.N., Anikina M.A., Platonov I.A.
}

Samara National Research University, Samara

\begin{abstract}
The analysis of exhaled breath is an actively developing area of non-invasive medical diagnostics. This approach helps to study the dynamics of physiological processes in the human body and detect pathologies at early stages of their development. However, the approach is not widely used due to the imperfections in sampling and sample preparation techniques leading to significant errors in the obtained results.

The aim of our study was to develop devices, methods, and a methodology that would enhance the accuracy of the non-invasive quantitative determination of acetone in exhaled breath used in non-invasive diagnostics of diabetes mellitus. The object of our study were devices which, when used during the calibration and preparation of samples, reduce the overall error of the analysis.

We used medical needles filled with a sorbent as containers for the chromato-desorption microsystem (CDmS). The sorbents were an inert carrier Chromaton N-AW modified with $25 \%$ sorption-active inorganic salt $\mathrm{CoCl}_{2}$, and an intert fibre covered with $25 \%$ polyethylene glycol (PEG-20M). The CDmS was saturated in the flow and drop regimes at the temperatures of 25 and $50^{\circ} \mathrm{C}$. The desorption of the analyte was performed at 70 and $100^{\circ} \mathrm{C}$ by means of discrete blowing with a $0.5 \mathrm{ml}$ inert gas (nitrogen) through the $\mathrm{CDmS}$ placed in a thermal desorption unit connected to the vaporiser of a gas chromatograph.

The results of the study of the prepared CDmS demonstrated the advantages of the drop method over the flow saturation, since it allows a greater number of discrete inputs within a single cycle and thus ensures a constant concentration of the analyte in the gas mixture. The article demonstrates that by regulating the conditions for the preliminary preparation of the system (the choice of the saturation and desorption technique) we can obtain gas mixtures with preset concentrations of acetone. The article also proves the possibility of using multiple-point calibration when using CDmS. Various concentrations of calibration gas mixtures can be obtained by changing the desorption temperature and by regulating the conditions for the preliminary preparation of the system, i.e. the amount of the inert gas or acetone gas mixture. The experiments demonstrated that the optimal method of $\mathrm{CDmS}$ saturation is the drop method (with $1 \mu 1$ dosage), while the preliminary preparation of the systems should be performed discretely at $25^{\circ} \mathrm{C}$ at the speed of $0.5 \mathrm{ml} / \mathrm{min}$. The desorption temperature may vary from 70 to $100^{\circ} \mathrm{C}$ while the blowing time depends on the desorption stage. When using systems with $\mathrm{CoCl}_{2}$ it is inadvisable to set the temperature above $100^{\circ} \mathrm{C}$.

The experiments demonstrated that with discrete dosing the stability error $\Delta$ of the obtained gas mixtures varies from 10 to $15 \%$, which enhances the accuracy of the analysis performed using the devices with CDmS. The obtained dependencies and developed techniques can be used to design a device for express quantitative determination of acetone in exhaled breath and to increase its sensitivity to this biomarker as well as to others.
\end{abstract}

Keywords: gas chromatography, non-invasive diagnostics, biomarkers, chromato-desorption microsystems, concentration.

\section{References}

1 World Health Organization. Global report on diabetes. Geneva, 2018, 88 p.

2 Horvath I., Barnes P.J., Loukides S., Sterk P.J. et al., European Respiratory Journal, 2017,
Vol. 49, No 4, pp. 1600965. DOI: 10.1183 / 13993003. 00965-2016

3 Su M., Ge L., Kong Q., Zheng X. et al., Biosensors and Bioelectronics, 2015, Vol. 63, pp. 232-239. DOI:10.1016/j.bios.2014.07.046 
4 Barberis A., Garbetta A., Cardinali A., Bazzu G. et al., Biosensors and Bioelectronics, 2017, Vol. 88, pp. 159-166. DOI:10.1016/j.bios.2016.08.007

5 Chung J.-H., Lee KE, Nam C.-W., Doh J.H. et al., The American Journal of Cardiology, 2017, Vol. 120, No 3, pp. 362-368. DOI: 10.1016 / j.amjcard.2017.04.057

6 Phillips M., Scientific American, 1992, Vol. 267, No 3, pp. 74-79.

7 Vax V.L., Domracheva E.G., Sobakinskaya E.A., Chernyaeva M.B., Advances in physical sciences. Devices and research methods, 2014, Vol. 184, No 7, pp. 739-758.

8 Soury S., Bahrami Ab., Alizadeh S., Shahna F.G. et al., Microchemical Journal, 2019, Vol. 784, No 148, pp. 346-354.

9 Heidari M., Bahrami Ab., Ghiasvand A.R., Shahna F.G. et al., Analytica Chimica Acta, 2013, Vol. 126, No 785, pp. 67-74.

10 Zhuravleva G.A. Diss. cand. chem. nauk. St. Petersburg, 2014, 102 p.

11 Lurie A.A., Chromatographic materials, M., Chemistry Publ., 1978, 440 p.

12 Platonov I.A., Kolesnichenko I.N., Platonov V.I., Lobanova M.S. et al., Modern sci-

Колесниченко Ирина Николаевна - к.х.н., доцент кафедры химии самарского национальноисследовательского университета имени академика С.П. Королева, Самара

Аникина Мария Андреевна - студент, самарского национально-исследовательского университета имени академика С.П. Королева, Самара

Платонов Игорь Артемьевич - д.т.н., професcop кафедры химии самарского национальноисследовательского университета имени академика С.П. Королева, Самара ence: actual problems and solutions, 2016, No 1, pp. 41-46.

13 Platonov I.A., Kolesnichenko I.N., Novikova E.A., Pavlova L.V. et al., Measuring technique, 2017, No 8, pp. 67-70.

14 Forina M., Annali di Chemica, 1975, Vol. 65, pp. 491-508.

15 Vitenberg A.G., Journal of Chromatography, 1991, Vol. 556, pp. 1-24.

16 Platonov I.A., Kolesnichenko I.N., Lange P.K., Measurement Techniques, 2017, Vol. 59, No 12, pp. 1330-1333.

17 Berezkin V.G., Platonov I.A., Smygina I.N., Chemistry and chemical technology, 2007, Vol. 50, No 8, pp. 22-24.

18 Platonov I.A., Ismagilov D.R., Kudryashov S.Yu., Smygina I.N. et al., Journal of analytical chemistry, 2006, Vol. 61, No 1. pp. 59-64.

19 Wittenberg A.G., Konopelko L.A., Journal of analytical chemistry, 2011, Vol. 66, No 5, pp. 452-472.

20 Platonov I.A., Sorptsionnye I khromatograficheskiye protsessy, 2006, Vol. 6, No 5, pp. 833-843.

21 Forina M., Lanteri S., Casale M., Journal of Chromatography A, 2007, Vol. 1158, pp. 6193.

Kolesnichenko Irina Nikolaevna - $\mathrm{PhD}$ in Chemistry, senior lecturer of Department of Chemistry, Samara National Research University, Samara, email: irniks@mail.ru

Anikina Maria Andreevna - student, Samara National Research University, Samara, e-mail: mariaanikina2502@mail.ru

Platonov Igor Artemyevitch - Candidate of Engineering Sciences, professor of Department of Chemistry, Samara National Research University, Samara, e-mail:pia@ssau.ru 